\title{
LncSOX4 serves an oncogenic role in the tumorigenesis of epithelial ovarian cancer by promoting cell proliferation and inhibiting apoptosis
}

\author{
YULAN LIU ${ }^{1}$, YAN WANG ${ }^{1}$, DONGMEI YAO ${ }^{1}$ and DIANSHENG CUI ${ }^{2}$ \\ ${ }^{1}$ Gynecology Department, Hubei Women and Children's Hospital, Wuhan, Hubei 430070; \\ ${ }^{2}$ Urology Department, Hubei Cancer Hospital, Wuhan, Hubei 430079, P.R. China
}

Received September 17, 2017; Accepted March 6, 2018

DOI: $10.3892 / \mathrm{mmr} .2018 .8892$

\begin{abstract}
Epithelial ovarian cancer is one of the primary causes of gynecological cancer mortality. Increasing evidence has suggested that long non-coding RNAs (IncRNAs) may serve a pivotal role in cancer development. To determine whether Lnc SRY-box 4 (SOX4), an lncRNA, promotes the self-renewal of liver tumor cells and contributes to the development of epithelial ovarian cancer, the present study investigated the expression of LncSOX4 in clinical epithelial ovarian cancer tissues and non-cancer controls by reverse transcription-quantitative polymerase chain reaction analysis. In addition, siRNA targeting LncSOX4 was designed and transfected into epithelial ovarian cancer cells to further assess the effect of knocking out LncSOX4 on cellular apoptosis, cell viability, proliferation and the cell cycle. The results demonstrated that the LncSOX4 expression level was significantly upregulated in epithelial ovarian cancer tissues (3.98 vs. 1.71, P<0.001). Silencing LncSOX4 in the SKOV3 and OVCAR3 cell lines significantly impaired cell proliferation $(\mathrm{P}<0.001)$. Cell cycle assays revealed that the proportion of cells in the G0/G1 phase increased significantly, whereas those in the $\mathrm{S}$ phase and $\mathrm{G} 2 / \mathrm{M}$ phase decreased. Apoptosis rate additionally increased following knockdown of LncSOX4 in the two cell lines. Furthermore, it was observed that an increased LncSOX4 expression level was positively associated with larger tumor sizes, more advanced tumor grade and more distant metastases.
\end{abstract}

\section{Introduction}

Epithelial ovarian cancer (EOC) is the leading cause of mortality from gynecological malignancy. The majority of patients with EOC are at a late stage upon diagnosis and

Correspondence to: Dr Diansheng Cui, Urology Department, Hubei Cancer Hospital, 116 Zhuodaoquan South Road, Hongshan, Wuhan, Hubei 430079, P.R. China

E-mail: health_2005@163.com

Key words: long non-coding RNA SRY-box 4, epithelial ovarian cancer the 5-year survival rate of EOC is $<30 \%$ (1-3). The standard therapy for EOC for current clinical application is surgery and platinum-based cytotoxic chemotherapy (4). The clinical management of EOC is confounded by the difficulty to accurately diagnose EOC at diagnosis. In addition, approximately $20 \%$ of patients will not respond to platinum based therapy and may relapse $(5,6)$.

Therefore, understanding the molecular mechanism underlying EOC is important for developing novel therapeutic targets. Long non-coding RNAs (lncRNAs) refer to RNAs having $>200$ nucleotides that cannot be transcribed into proteins. LncRNAs are evolutionarily conserved and are expressed in a tissue specific manner (7). They are involved in various cellular processes and their dysregulation is emerging as important factors in tumor development and therapy response (8-10). IncRNAs have been reported to act as oncogenes or tumor suppressors in cancer development $(11,12)$. A recent study demonstrated that Lnc SRY-box 4 (SOX4) was able to promote the self-renewal of liver tumor-initiating cells through signal transducer and activator of transcription 3-mediated Sox4 expression and may serve as an oncogene in liver cancer (13). Furthermore, it has been reported that LncSOX4 expression is associated with relapse rate and poor outcome in EOC (14). The present study investigated the role of LncSOX4 in EOC. LncSOX4 expression was significantly upregulated in patients with EOC and in EOC cell lines. Knocking out LncSOX4 resulted in inhibition of tumor cell growth, migratory ability and cell proliferation. The cell cycle was arrested at the G0/G1 phase following LncSOX4 silencing. In addition, the LncSOX4 expression level was additionally associated with unfavorable clinical prognostic factors. It was observed that LncSOX4 may serve as an oncogene in EOC and that downregulating its expression may be a potential therapeutic target.

\section{Materials and methods}

Patients and specimens. Ovarian tissues from 30 female

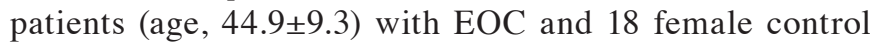
patients (age, 46.2 \pm 12.4 ) were collected between April 2015 and April 2017 from Hubei Women and Children's Hospital (Wuhan, China). The study was approved by the Research 
Ethics Committee of Hubei Women and Children's Hospital. All selected patients provided written informed consent. Patients were sorted into a high LncSOX4 expression group and a low LncSOX4 expression group, according to the median LncSOX4 expression level (Median relative expression, 3.99). The diagnosis of EOC was based on the clinical presentation, morphological criteria and immunohistochemical staining. Patients who had received radiation, chemotherapy or hormonal therapy were excluded from the present study. Information about the patients is presented in Table I.

Cell culture and culture conditions. EOC cell lines including SKOV3, HO8910-PM, OVCAR3 and the human ovarian immortalized non-tumorigenic ovarian surface epithelial (IOSE-80) cell line were purchased from the American Type Culture Collection (Manassas, VA, USA). The high grade ovarian serous adenocarcinoma HEY-A8 cell line was provided by Professor Zehua Wang from the Wuhan Union Hospital (Wuhan, China). Cells were cultured in RPM1-1640 medium (Gibco; Thermo Fisher Scientific, Inc., Waltham, MA, USA) containing 10\% fetal bovine serum (FBS; Gibco; Thermo Fisher Scientific, Inc.) and maintained at $37^{\circ} \mathrm{C}$ in a $5 \% \mathrm{CO}_{2}$ incubator.

Cell transfection. The SKOV3 cells (1x $10^{6} /$ well) and OVCAR3 cells ( $1 \times 10^{6} /$ well) were transfected with small interfering (si)-LncSOX4 and the negative control (NC) using Lipofectamine ${ }^{\circledR} 3000$ (Thermo Fisher Scientific, Inc.). si-LncSOX4 and NC were purchased from Shanghai GenePharma Co., Ltd. (Shanghai, China). The expression level of LncSOX4 was detected by reverse transcription-quantitative polymerase chain reaction (RT-qPCR) analysis according to the protocol detailed below in order to determine transfection efficiency. The sequence of si-LncSOX4 was 5'-GGA TGACAAGAAGTACAAA-3. The sequence of the negative control was: Sense, 5'-UUCUCCGAACGUGUCACGUTT-3' and antisense, 5'-ACGUGACACGUUCGGAGAATT-3'. Cells were incubated for $48 \mathrm{~h}$ after transfection and then used for further analysis.

RT-qPCR analysis. Total RNA was extracted from human samples and ovarian cell lines using TRIzol (Invitrogen; Thermo Fisher Scientific, Inc.). A reverse transcription kit (Applied Biosystems; Thermo Fisher Scientific, Inc.) was used to transform RNA into cDNA. The RT reaction was performed at $37^{\circ} \mathrm{C}$ for $15 \mathrm{~min}$ and $85^{\circ} \mathrm{C}$ for $5 \mathrm{sec}$. The level of LncSOX 4 was measured using a SYBR Premix kit (Takara420a; Takara Bio, Inc., Otsu, Japan) using GAPDH as the endogenous control. The qPCR reaction was performed in an Applied Biosystems 7500 Fast Real-Time PCR system (Thermo Fisher Scientific, Inc.) at $95^{\circ} \mathrm{C}$ for $3 \mathrm{~min}$, followed by 40 cycles at $95^{\circ} \mathrm{C}$ for $15 \mathrm{sec}$, and finally $60^{\circ} \mathrm{C}$ for $1 \mathrm{~min}$. The relative expression of LncSOX4 was calculated using the comparative $\mathrm{Cq}\left(2^{-\Delta \Delta \mathrm{Cq}}\right)$ method (15). The primers for LncSOX4 included (forward) 5'-AGCGACAAGATCCCT TTCATTC-3' and (reverse) 5'-CGTTGCCGGACTTCACCTT-3'. The primers for GAPDH included (forward) 5'-GGCTGAGAACGGGAA GCTTGTCAT-3' and (reverse) 5'-CAGCCTTCTCCATGG TGGTGAAGA-3'.
In situ hybridization (ISH). ISH was performed using a ISH Detection Kit III (AP; Boster Biological Technology, Pleasanton, CA, USA) according to the manufacturer's protocol. Samples were digested with proteinase K for $5 \mathrm{~min}$, fixed in $4 \%$ paraformaldehyde for $1 \mathrm{~h}$ at room temperature and hybridized with a 5'-digoxin-labeled probe for LncSOX4 with a sequence of 5'-CATGGGATCTTACTACAGGA-3', at $55^{\circ} \mathrm{C}$ overnight. Paraffin-embedded sections of $7 \mu \mathrm{m}$ thickness were deparaffinized with xylene and washed with ethanol at concentrations of 100, 95, 85 and 75\%. Samples were incubated with horseradish peroxidase at $4^{\circ} \mathrm{C}$ for $30 \mathrm{~min}$. The results were observed using a light microscope (magnification, $\mathrm{x} 200$ ) following the addition of hematoxylin at $15^{\circ} \mathrm{C}$ for $5 \mathrm{~min}$ and eosin at $15^{\circ} \mathrm{C}$ for $2 \mathrm{~min}$.

Transwell assay. Following $48 \mathrm{~h}$ of transfection, SKOV3 cells were harvested using RPMI-1640 without serum. $150 \mu \mathrm{l}$ cell suspension $\left(2.5 \times 10^{4}\right.$ cells) was added into the upper chamber (Corning Incorporated, Corning, NY, USA) and $600 \mu \mathrm{l}$ culture medium containing $10 \%$ FBS was added into the lower chamber. The membrane was pre-coated with Matrigel (Corning Incorporated) for $6 \mathrm{~h}$ prior to the invasion assay. Following incubation for $12 \mathrm{~h}$, the cells were fixed with methanol for $10 \mathrm{~min}$ at $4^{\circ} \mathrm{C}$ and stained with $0.1 \%$ crystal violet at room temperature for $5 \mathrm{~min}$. The results were captured using an inverted light microscope (magnification, x200; TS100; Nikon Corporation, Tokyo, Japan).

Cell proliferation assay. The cell proliferation assay was carried out using a Cell Counting Kit-8 (CCK-8; Dojindo Molecular Technologies, Inc., Kumamoto, Japan), according to the manufacturer's protocol. SKOV3 and OVCAR3 cells were seeded into 96-well plates, with each well containing 3,000-5,000 cells. Cells were incubated for 24, 48 and $72 \mathrm{~h}$, respectively. Subsequently, $10 \mu 1$ CCK- 8 was added to the wells and the optical density was read at $450 \mathrm{~nm}$ with a microplate reader once every $30 \mathrm{~min}$ for $4 \mathrm{~h}$ in total.

Western blotting. Following 48 h of si-LncSOX4 and NC transfection, cells were lysed on ice in radioimmunoprecipitation assay lysis buffer (Beyotime Institute of Biotechnology, Haimen, China). A bicinchoninic acid protein assay kit (Goodbio Biotechnology Co., Ltd., Wuhan, China) was used for protein determination. Protein samples of $20 \mu \mathrm{g} /$ lane were separated on 8-12\% SDS-PAGE gels (Goodbio Biotechnology Co., Ltd.) and transferred onto nitrocellulose membranes. Membranes were blocked in Tris-buffered saline with $0.05 \%$ Tween-20 and 5\% non-fat milk for $1 \mathrm{~h}$ at $37^{\circ} \mathrm{C}$. Membranes were subsequently incubated with antibodies against $72 \mathrm{kDa}$ type IV collagenase (MMP2; cat. no. ab37150; 1:1,000; Abcam, Cambridge, UK), matrix metalloproteinase 9 (MMP9; cat. no. ab38898; 1:1,000; Abcam) and GAPDH (cat. no. ab9485; 1:2,500; Abcam) overnight at $4^{\circ} \mathrm{C}$. Immunodetection of the bound antibodies was conducted using a goat anti-rabbit immunoglobulin $\mathrm{G}$ horseradish peroxidase conjugated antibody (cat. no. 7074; 1:2,000; Cell Signaling Technology, Inc., Danvers, MA, USA) for $1 \mathrm{~h}$ at room temperature. Immunostaining was detected using an enhanced chemiluminescence substrate (Beyotime Institute of Biotechnology) and signal intensities were determined 
Table I. Association between LncSOX4 expression levels and clinical data in 30 patients with epithelial ovarian cancer.

\begin{tabular}{|c|c|c|c|c|}
\hline \multirow[b]{2}{*}{ Variable } & \multirow[b]{2}{*}{ No. patients $(n=30)$} & \multicolumn{2}{|c|}{ LncSOX4 expression level } & \multirow[b]{2}{*}{ P-value } \\
\hline & & Low $(n=15)$ & High $(n=15)$ & \\
\hline \multicolumn{5}{|l|}{ Age } \\
\hline$>55$ & 17 & 7 & 10 & 0.461 \\
\hline$\leq 55$ & 13 & 8 & 5 & \\
\hline \multicolumn{5}{|c|}{ FIGO stage } \\
\hline I-II & 14 & 12 & 2 & 0.001 \\
\hline III-IV & 16 & 3 & 13 & \\
\hline \multicolumn{5}{|c|}{$\mathrm{CA} 125, \mathrm{U} / \mathrm{ml}$} \\
\hline$\leq 35$ & 17 & 10 & 6 & 0.272 \\
\hline$>35$ & 13 & 5 & 9 & \\
\hline \multicolumn{5}{|c|}{ Tumor size, $\mathrm{cm}$} \\
\hline$\leq 5$ & 14 & 11 & 3 & 0.010 \\
\hline$>5$ & 16 & 4 & 12 & \\
\hline \multicolumn{5}{|c|}{ Distant metastases } \\
\hline Yes & 18 & 3 & 10 & 0.027 \\
\hline No & 12 & 12 & 5 & \\
\hline
\end{tabular}

LncSOX4, long non-coding RNA SRY-box 4; FIGO, International Federation of Gynecology and Obstetrics.

using Quantity One image software (version 4.6.8; Bio-Rad Laboratories, Inc., Hercules, CA, USA).

Flow cytometric analysis. Following transfection with si-LncSOX4 or NC for $48 \mathrm{~h}$, SKOV3 and OVCAR3 cells were harvested for flow cytometric analysis. Cells were stained with the Annexin V-Fluorescein Isothiocyanate/Propidium Iodide Apoptosis Detection kit (cat. no. 556547; BD Biosciences, Franklin Lakes, NJ, USA), according to the manufacturer's protocol. Subsequently, the cell cycle was detected using a FACSCanto II (BD Biosciences) and analyzed using FlowJo software (version 7.6.1; FlowJo LLC, Ashland, OR, USA).

Statistical analysis. The data obtained are expressed as the mean \pm standard deviation. Two-way analysis of variance was performed when comparing continuous variables between groups, and Scheffe's post hoc test was performed where significant differences were observed. A $\chi^{2}$ test was used to compare enumerated data. Each experiment was repeated in triplicate. All analyses were performed using R Bioconductor (version 3.3.2; https://www.bioconductor.org/). $\mathrm{P}<0.05$ was considered to indicate a statistically significant difference.

\section{Results}

LncSOX4 is upregulated in EOC tissues and cell lines. The expression levels of LncSOX4 in the ovarian tissues of 30 patients with EOC and 18 control patients were detected via RT-qPCR. Relative LncSOX4 expression levels in patients with EOC were significantly upregulated compared with non-tumor samples $(3.98$ vs. $1.71 ; \mathrm{P}<0.001)$, the results of which are presented in Fig. 1A. To further confirm these results, LncSOX4 expression levels were detected in the EOC cell lines HO8910-PM, SKOV-3, HEY-A8 and OVCAR3 and the non-tumor cell line IOSE-80. Significant differences in LncSOX4 expression levels were observed between these five groups $(\mathrm{P}<0.05)$. The results demonstrated that the expression levels of LncSOX4 in EOC cell lines were significantly higher compared with IOSE-80 (HO8910-PM vs. IOSE80, $\mathrm{P}<0.001$; SKOV-3 vs. IOSE80, $\mathrm{P}<0.001$; HEY-A8 vs. IOSE80, $\mathrm{P}<0.001$; OVCAR-3 vs. IOSE80, $\mathrm{P}<0.001$; Fig. 1B). A representative EOC specimen and surrounding non-tumor tissue was used for ISH analysis, and elevated expression levels of LncSOX4 were observed only in EOC tumor cells (Fig. 1C).

Knocking out LncSOX4 inhibits cell proliferation in EOC cell lines. To further study the function of LncSOX4 in EOC, siRNA targeting LncSOX4 was transfected to knock out LncSOX4 from EOC cell lines. SKOV-3 and OVCAR3 were used for transfection and further analysis. The transcription levels of LncSOX4 were decreased by 58 and $69 \%$ following transfection with si-LncSOX4 in SKOV-3 and OVCAR3 cells, respectively (si-LncSOX4 vs. Control, $\mathrm{P}<0.001$; si-LncSOX4 vs. NC, $\mathrm{P}<0.001$; si-LncSOX4 vs. Control, $\mathrm{P}<0.001$; si-LncSOX4 vs. NC, P<0.001; Fig. 2), which confirmed the high efficiency of si-LncSOX4. CCK-8 assays were performed following transfection. In the first $72 \mathrm{~h}$, the cell proliferation rate did not exhibit significant differences between the SKOV-3, NC and si-LncSOX4 groups, according to the results of analysis of variance $(\mathrm{P}>0.05)$. Significant inhibition of cell proliferation was observed following $72 \mathrm{~h}$ between the three groups $(\mathrm{P}<0.05)$ : Day 4, SKOV-3 vs. NC, 2.7 vs. 4.1, $\mathrm{P}=0.013$; OVCAR3 vs. NC, 3.2 vs. 4.3, $\mathrm{P}=0.007$; Day 5, SKOV-3 vs. NC, 3.8 vs. 7.1, $\mathrm{P}<0.001$; OVCAR3 vs. $\mathrm{NC}, 7.2$ vs. 4.3, $\mathrm{P}<0.001$; Fig. 3), which indicated that LncSOX4 promoted cell proliferation and acted as an oncogene in EOC. 
A

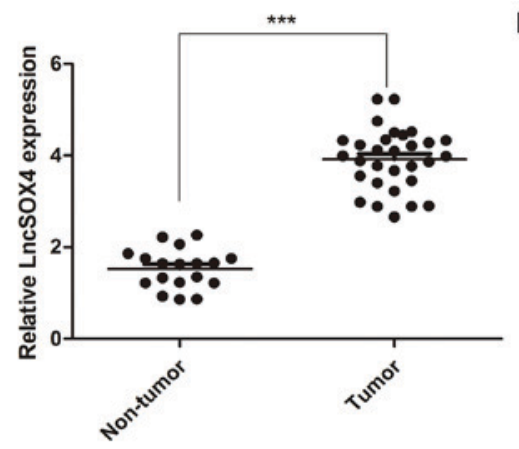

C

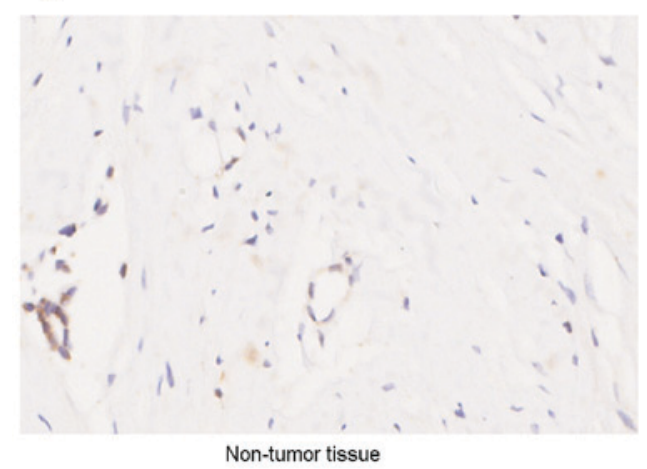

B

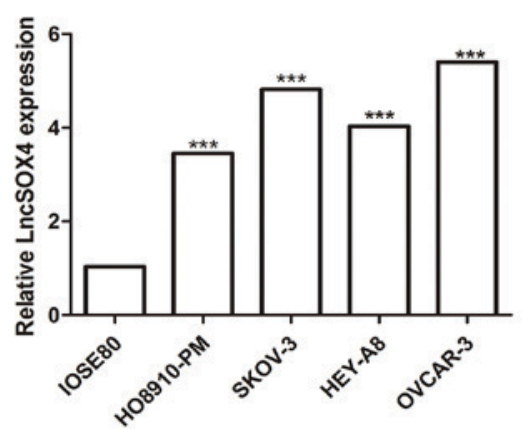

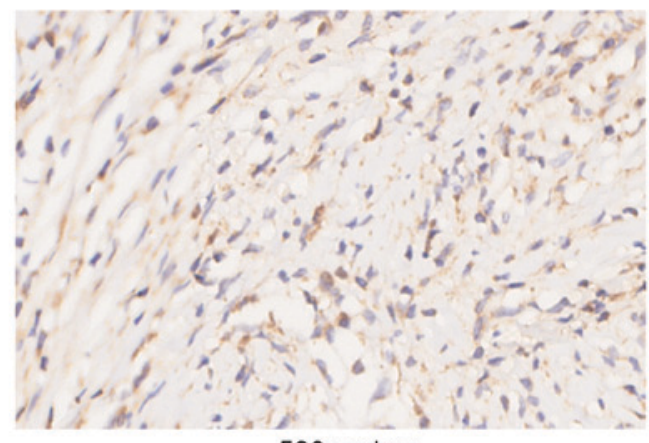

EOC specimen

Figure 1. Level of LncSOX4 in EOC tumor tissues and non-tumor controls. The transcription level of LncSOX4 was significantly upregulated in (A) EOC tumor tissues and (B) EOC cell lines compared with non-tumor IOSE80 cells. ${ }^{* * * *} \mathrm{P}<0.001$ vs. respective control. (C) In situ hybridization demonstrated elevated levels of LncSOX4 only in EOC tumor cells. Magnification, x200. EOC, epithelial ovarian cancer; LncSOX4, long non-coding RNA SRY-box 4.
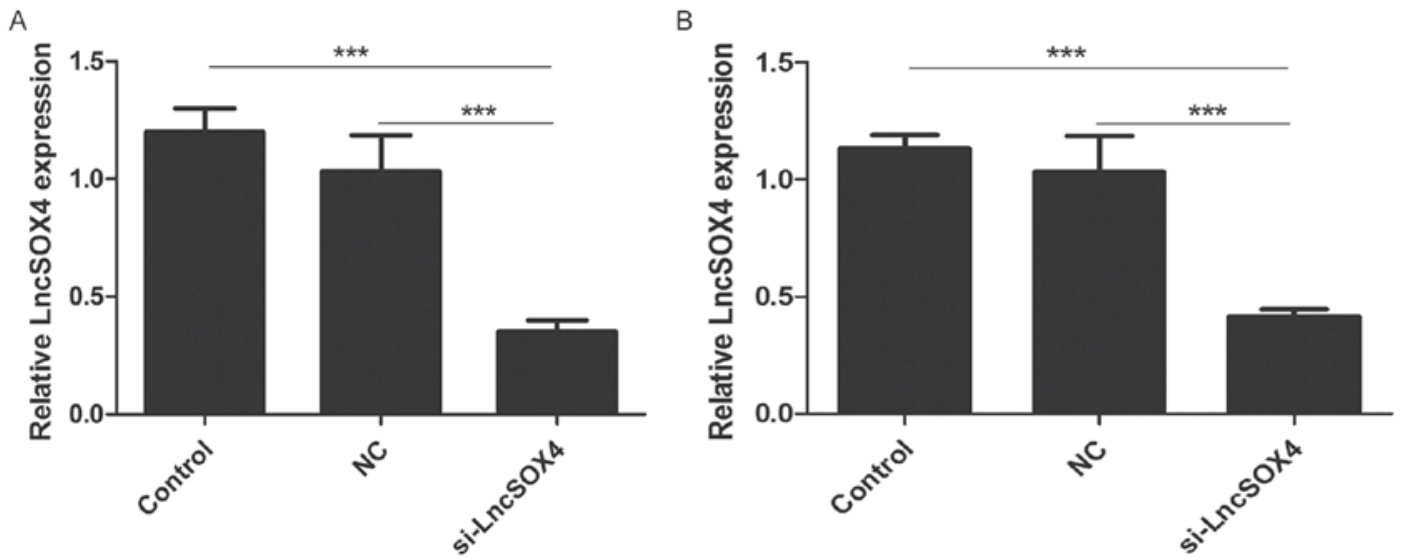

Figure 2. Relative LncSOX4 expression levels in EOC cell lines. LncSOX4 expression levels in (A) SKOV-3 and (B) OVCAR-3 cells were downregulated following transfection with si-LncSOX4. ${ }^{* * *} \mathrm{P}<0.001$. LncSOX4, long non-coding RNA SRY-box 4; NC, negative control; si, small interfering.

A

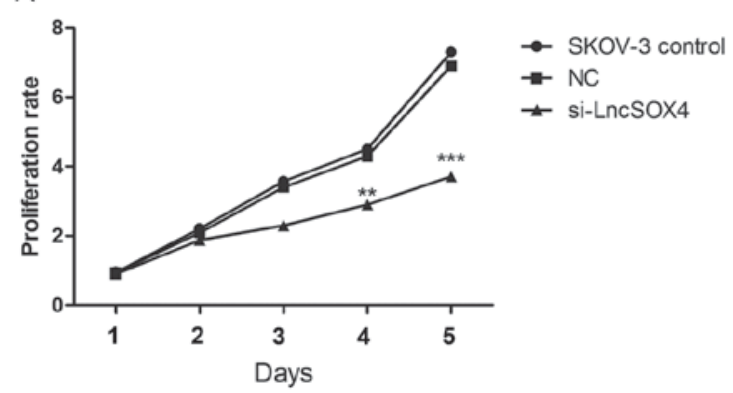

B

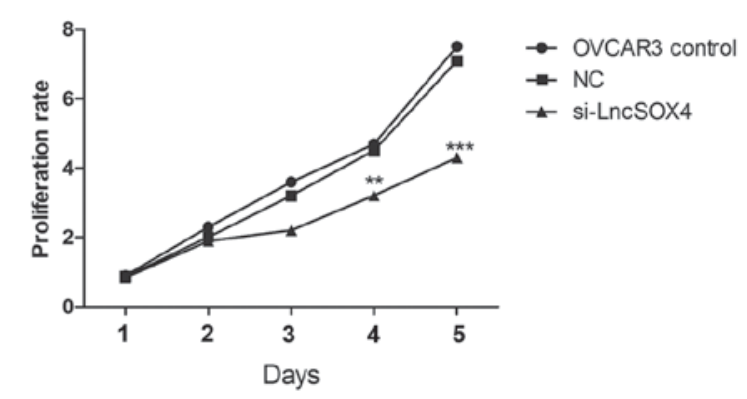

Figure 3. Proliferation of EOC cell lines following LncSOX4 silencing. (A) SKOV-3 and (B) OVCAR-3 cell proliferation was inhibited by knocking down LncSOX4. ${ }^{* *} \mathrm{P}<0.01,{ }^{* * *} \mathrm{P}<0.001$ vs. respective control. LncSOX4, long non-coding RNA SRY-box 4; NC, negative control; si, small interfering. 
A
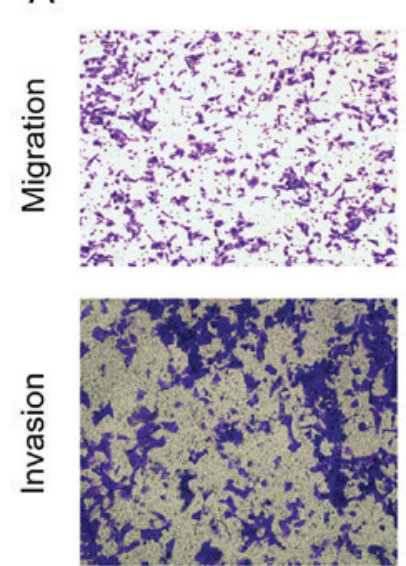

si-LncSOX4

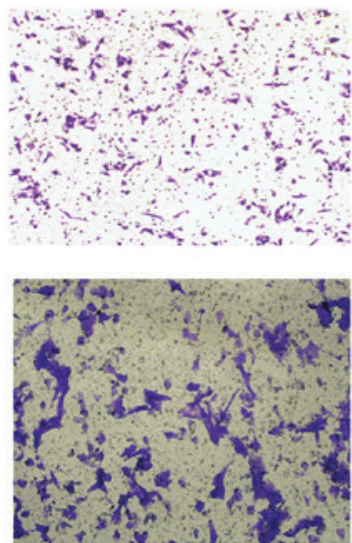

B

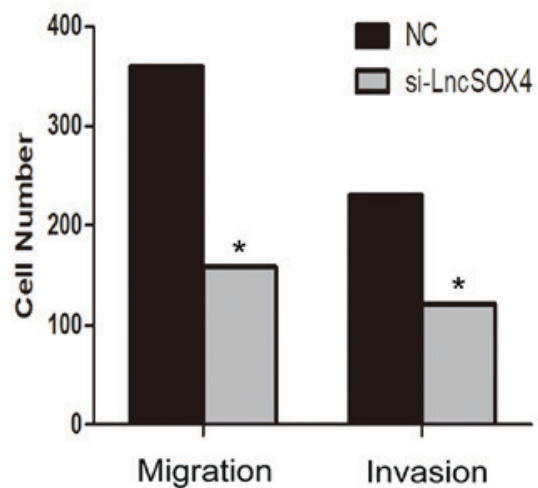

Figure 4. Knockdown LncSOX4 inhibits the migration and invasion of SKOV-3 cells. (A) SKOV-3 cell migration and invasion ability decreases following silencing of LncSOX. Magnification, x200. (B) Quantification of the migrated and invaded SKOV-3 cells. * P<0.05 vs negative control. LncSOX4, long non-coding RNA SRY-box 4; NC, negative control; si, small interfering.
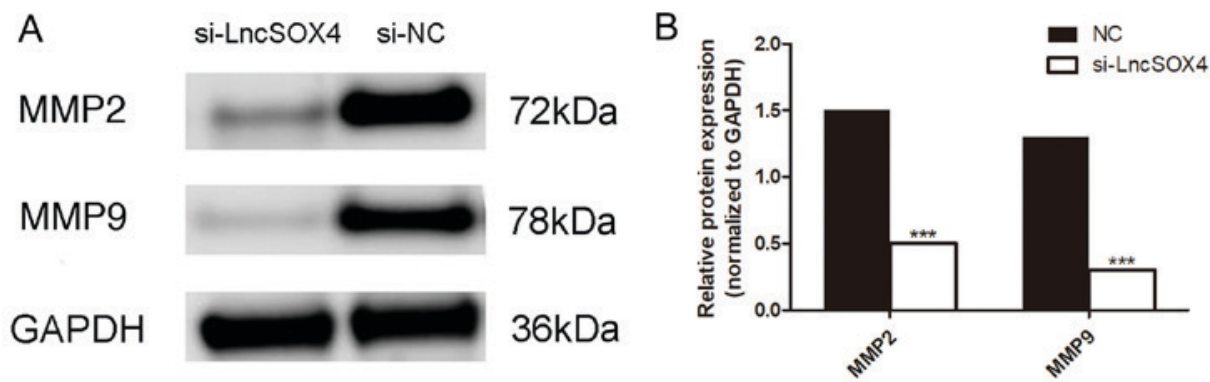

Figure 5. Expression of MMP2 and MMP9 is downregulated in the si-LncSOX4 group compared with the NC group. (A) Western blot analysis was used to determine protein expression and (B) densitometric analysis was performed to quantify the results. ${ }^{* * * *} \mathrm{P}<0.001$. MMP2, $72 \mathrm{kDa}$ type IV collagenase; MMP9, matrix metalloproteinase 9; LncSOX4, long non-coding RNA SRY-box 4; NC, negative control; si, small interfering.
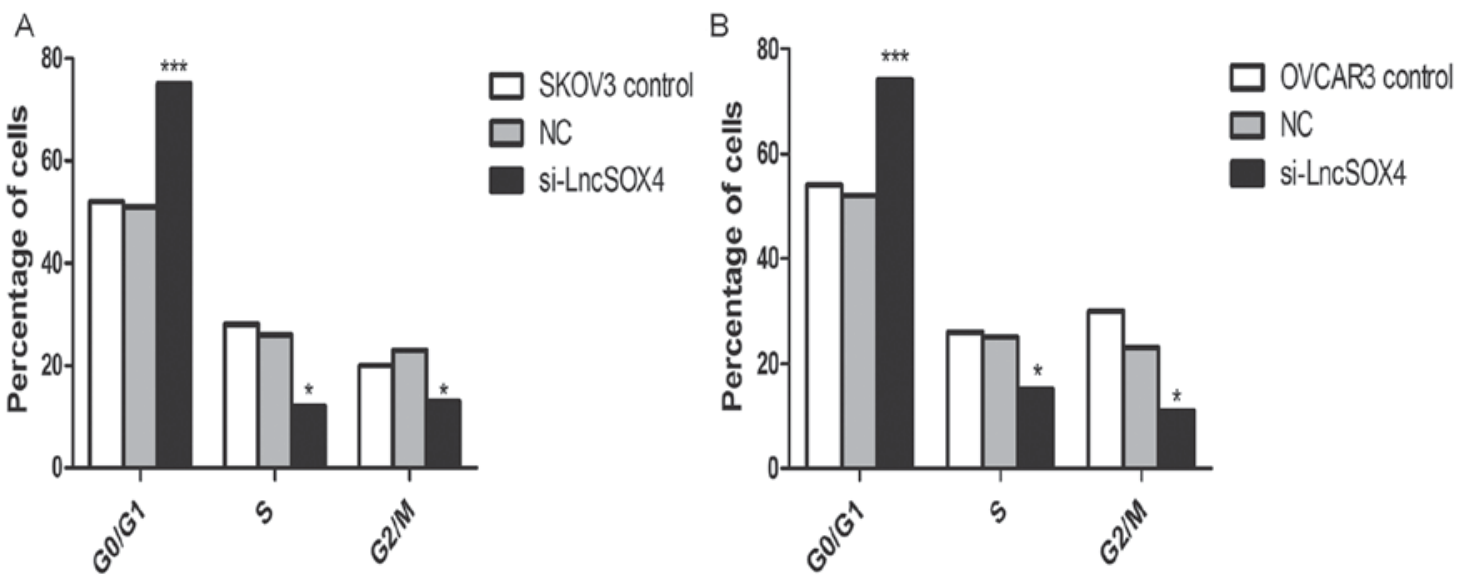

Figure 6. Cell cycle analysis of EOC cells following LncSOX4 silencing. (A) The SKOV-3 and (B) OVCAR-3 cell cycle was arrested at the G0/G1 phase following LncSOX4 knockdown, while the number of cells in the $\mathrm{S}$ and $\mathrm{G} 2 / \mathrm{M}$ phases decreased. ${ }^{*} \mathrm{P}<0.05$ and ${ }^{* * * *} \mathrm{P}<0.001$ vs. respective control. LncSOX4, long non-coding RNA SRY-box 4; NC, negative control; si, small interfering.

Knocking out LncSOX4 inhibits migration and invasion in the SKOV3 cell line. A Transwell assay was performed to observe the role of LncSOX4 in the SKOV3 cell line. Migratory and invasive abilities were visually decreased following transfection with si-LncSOX4 (Fig. 4A). Migratory ability decreased by up to $56 \%$ in the SKOV3 cell line and invasive ability was inhibited by up to $47 \%$ (Fig. 4B). In addition, the protein expression levels of MMP2 and MMP9 were assessed in the si-LncSOX4 and NC groups in the SKOV3 cell line (Fig. 5A). Consistent with the results of the Transwell assay, decreased expression of MMP2 and MMP9 was identified in the si-LncSOX4 group (Fig. 5B), indicating that LncSOX4 may 
upregulate MMP2 and MMP9 protein expression. These data suggested that LncSOX4 promoted cell migration and invasion in an ovarian cancer cell line.

Knocking out LncSOX4 arrests the cell cycle at the G0/G1 phase in EOC cell lines. The cell cycle was additionally analyzed in SKOV-3 and OVCAR3 cell lines following knockdown of LncSOX4. As presented in Fig. 6, the percentage of cells in the G0/G1 phase was significantly increased in the two cell lines compared with the NC groups (SKOV-3, 77\% vs. $50 \%, \mathrm{P}<0.001$; OVCAR3, $73 \%$ vs. $52 \%, \mathrm{P}<0.001)$. In addition, the proportions of cells in $\mathrm{S}$ and $\mathrm{G} 2 / \mathrm{M}$ phases decreased significantly in SKOV-3 and OVCAR3 cells $(\mathrm{P}<0.05)$.

LncSOX4 expression is associated with unfavorable prognostic factors in patients with EOC. The cell experiments indicated that LncSOX4 may serve an oncogenic role in EOC cell lines. To assess whether the expression of LncSOX4 was associated with clinicopathological parameters, a comparative study was performed by sorting patients into a high and low LncSOX4 expression group, according to the median LncSOX4 expression level of 3.99. Table I summarizes the association between LncSOX4 expression and clinical parameters. It was demonstrated that the expression level of LncSOX4 was positively associated with International Federation of Gynecology and Obstetrics stage (16), tumor size and distant metastases, with P-values of $0.000,0.010$ and 0.027 , respectively, and not associated with CA125 levels.

\section{Discussion}

Epithelial ovarian cancer, as the commonest type of ovarian cancer, usually occurs in women $>50$ years old. Among all cancer types in female patients, ovarian cancer has the highest mortality rate, and the majority of patients with EOC are diagnosed at a late stage due to its nonspecific signs and symptoms $(17,18)$. As a result, the 5-year survival rate of EOC is $<30 \%$ (19). An increasing number of studies have been conducted to elucidate the pathogenesis of this disease and to identify circulating biomarkers for the early detection of EOC (20-24).

lncRNAs consist of $>200$ nucleotides and have a limited protein-coding capacity. IncRNAs have been reported to have important effects on cell proliferation, apoptosis and differentiation of various kinds of human cancer (25-27), including EOC $(28,29)$. LncSOX4 was first reported to promote liver cancer progression (13). To determine whether this lncRNA additionally served an oncogenic role in the tumorigenesis of EOC, relevant experiments were performed in the present study, and it was identified that the transcription levels of LncSOX4 were significantly upregulated in EOC tissues and cell lines compared with non-tumor controls. In addition, IncRNAs may promote cell proliferation, migration and invasion. Cell cycle analysis additionally demonstrated that inhibition of LncSOX4 was able to arrest the cell cycle at the G0/G1 phase.

Deficiencies of diagnostic and prognostic factors are responsible for the poor outcome of ovarian cancer. A number of studies have been conducted to identify efficient serological biomarkers of ovarian cancer (30-33). However, there is no reliable biomarker at present. Recently, lncRNAs have been analyzed in various studies on EOC, and certain among them may have prognostic effects (14). It was reported that lncSOX4 was associated with relapse and poor outcomes in EOC (14). The results of the present study are consistent with previous findings that the high expression level of LncSOX4 was associated with unfavorable clinical prognostic factors (14). This indicated that LncSOX4 may serve as an unfavorable biomarker for EOC patients.

The present study investigated the role of LncSOX4 in EOC cell lines in vitro, although the molecular mechanism remains unknown. It was previously reported that Pvt1 oncogene (PVT1), an lncRNA which exhibits the potential to predict poor prognosis for patients with stage I EOC, was upregulated by transforming growth factor (TGF) $\beta 1$ in hepatocellular carcinoma (34). Martini et al (14) isolated the most highly correlated genes to perform computed pathway enrichment for LncSOX4, and used the microGraphite pipeline to identify circuits associated with LncRNAs. They reported that in LncSOX4 circuits the upregulation of TGF $\beta 1$ was associated with poor prognosis for patients with EOC, suggesting a potential molecular association between PVT1 and LncSOX4.

In conclusion, LncSOX4 was upregulated in tissues from patients with EOC and EOC cell lines, and knocking out LncSOX4 was able to inhibit cell proliferation and arrest the cell cycle at the G0/G1 phase. Cellular migratory and invasive abilities decreased following transfection with si-LncSOX4. Following analysis of the clinical data, it was identified that LncSOX4 may serve as a novel biomarker and therapeutic target for EOC.

\section{Acknowledgements}

Not applicable.

\section{Funding}

No funding was received.

\section{Availability of data and materials}

All data generated or analyzed during this study are included in this published article.

\section{Authors' contributions}

YL and DC designed the study. YL prepared the manuscript and performed the statistical analysis. YW researched the literature and contributed to manuscript revisions. YL and DY performed the experiments. DC critically revised and edited the manuscript.

\section{Ethics approval and consent to participate}

The present study was approved by the Research Ethics Committee of Hubei Women and Children's Hospital.

\section{Consent for publication}

The patients provided written informed consent for the publication of associated data and accompanying images. 


\section{Competing interests}

The authors declare that they have no competing interests.

\section{References}

1. Clarke-Pearson DL: Clinical practice. Screening for ovarian cancer. N Engl J Med 361: 170-177, 2009.

2. Jemal A, Siegel R, Xu J and Ward E: Cancer statistics, 2010. CA Cancer J Clin 60: 277-300, 2010.

3. Diaz-Gil D, Fintelmann FJ, Molaei S, Elmi A, Hedgire SS and Harisinghani MG: Prediction of 5-year survival in advanced-stage ovarian cancer patients based on computed tomography peritoneal carcinomatosis index. Abdom Radiol (NY) 41: 2196-2202, 2016.

4. Pignata S, Cannella L, Leopardo D, Pisano C, Bruni GS and Facchini G: Chemotherapy in epithelial ovarian cancer. Cancer Lett 303: 73-83, 2011.

5. Kurman RJ and Shih IeM: The origin and pathogenesis of epithelial ovarian cancer: A proposed unifying theory. Am J Surg Pathol 34: 433-443, 2010.

6. Vaughan S, Coward JI, Bast RC Jr, Berchuck A, Berek JS, Brenton JD, Coukos G, Crum CC, Drapkin R, Etemadmoghadam D, et al: Rethinking ovarian cancer: Recommendations for improving outcomes. Nat Rev Cancer 11: 719-725, 2011.

7. Guttman M, Amit I, Garber M, French C, Lin MF, Feldser D, Huarte M, Zuk O, Carey BW, Cassady JP, et al: Chromatin signature reveals over a thousand highly conserved large non-coding RNAs in mammals. Nature 458: 223-227, 2009.

8. Mercer TR, Dinger ME and Mattick JS: Long non-coding RNAs: Insights into functions. Nat Rev Genet 10: 155-159, 2009.

9. Ponting CP, Oliver PL and Reik W: Evolution and functions of long noncoding RNAs. Cell 136: 629-641, 2009.

10. Huarte $M$ and Rinn JL: Large non-coding RNAs: Missing links in cancer? Hum Mol Genet 19: R152-R161, 2010.

11. Zhang J, Li Z, Liu L, Wang Q, Li S, Chen D, Hu Z, Yu T, Ding J, Li J, et al: Long noncoding RNA TSLNC8 is a tumor suppressor that inactivates the interleukin-6/STAT3 signaling pathway. Hepatology 67: 171-187, 2018.

12. Shih JW, Chiang WF, Wu ATH, Wu MH, Wang LY, Yu YL, Hung YW, Wang WC, Chu CY, Hung CL, et al: Long noncoding RNA LncHIFCAR/MIR31HG is a HIF-1 $\alpha$ co-activator driving oral cancer progression. Nat Commun 8: 15874, 2017.

13. Chen ZZ, Huang L, Wu YH, Zhai WJ, Zhu PP and Gao YF LncSox 4 promotes the self-renewal of liver tumour-initiating cells through Stat3-mediated Sox4 expression. Nat Commun 7: 12598, 2016.

14. Martini P, Paracchini L, Caratti G, Mello-Grand M, Fruscio R, Beltrame L, Calura E, Sales G, Ravaggi A, Bignotti E, et al: lncRNAs as novel indicators of patients' prognosis in stage I epithelial ovarian cancer: A retrospective and multicentric study. Clin Cancer Res 23: 2356-2366, 2017.

15. Livak KJ and Schmittgen TD: Analysis of relative gene expression data using real-time quantitative PCR and the 2(-Delta Delta C(T)) method. Methods 25: 402-408, 2001

16. Jayson GC, Kohn EC, Kitchener HC and Ledermann JA: Ovarian cancer. Lancet 384: 1376-1388, 2014.

17. Fujiwara K, McAlpine JN, Lheureux S, Matsumura N and Oza AM: Paradigm shift in the management strategy for epithelial ovarian cancer. Am Soc Clin Oncol Educ Book 35: e247-e257, 2016

18. Montagnana M, Benati M and Danese E: Circulating biomarkers in epithelial ovarian cancer diagnosis: From present to future perspective. Ann Transl Med 5: 276, 2017.

19. Holschneider $\mathrm{CH}$ and Berek JS: Ovarian cancer: Epidemiology, biology, and prognostic factors. Semin Surg Oncol 19: 3-10e, 2000 .
20. Sun X, Zhang W, Li H, Niu C, Ou Y, Song L and Zhang Y: Stonin 2 overexpression is correlated with unfavorable prognosis and tumor invasion in epithelial ovarian cancer. Int J Mol Sci 18: pii: E1653, 2017.

21. Yang X, Liang L, Zhang XF, Jia HL, Qin Y, Zhu XC, Gao XM, Qiao P, Zheng Y, Sheng YY, et al: MicroRNA-26a suppresses tumor growth and metastasis of human hepatocellular carcinoma by targeting interleukin-6-Stat 3 pathway. Hepatology 58: 158-170, 2013.

22. Bagnoli M, Canevari S, Califano D, Losito S, Maio MD, Raspagliesi F, Carcangiu ML, Toffoli G, Cecchin E, Sorio R, et al: Development and validation of a microRNA-based signature (MiROvaR) to predict early relapse or progression of epithelial ovarian cancer: A cohort study. Lancet Oncol 17: 1137-1146, 2016.

23. Walsh CS, Ogawa S, Karahashi H, Scoles DR, Pavelka JC, Tran H, Miller CW, Kawamata N, Ginther C, Dering J, et al: ERCC5 is a novel biomarker of ovarian cancer prognosis. J Clin Oncol 26: 2952-2958, 2008

24. Gagnon A and Ye B: Discovery and application of protein biomarkers for ovarian cancer. Curr Opin Obstet Gynecol 20: 9-13, 2008

25. Xing Z, Lin A, Li C, Liang K, Wang S, Liu Y, Park PK, Qin L, Wei Y, Hawke DH, et al: IncRNA directs cooperative epigenetic regulation downstream of chemokine signals. Cell 159: 1110-1125, 2014.

26. Trimarchi T, Bilal E, Ntziachristos P, Fabbri G, Dalla-Favera R, Tsirigos A and Aifantis I: Genome-wide mapping and characterization of Notch-regulated long noncoding RNAs in acute leukemia. Cell 158: 593-606, 2014.

27. Abraham JM and Meltzer SJ: Long noncoding RNAs in the pathogenesis of barrett's esophagus and esophageal carcinoma. Gastroenterology 153: 27-34, 2017.

28. Hua F, Li CH, Chen XG and Liu XP: Long noncoding RNA CCAT2 knockdown suppresses tumorous progression by sponging miR-424 in epithelial ovarian cancer. Oncol Res 26: 241-247, 2018.

29. Xiu YL, Sun KX, Chen X, Chen S, Zhao Y, Guo QG and Zong ZH: Upregulation of the lncRNA Meg3 induces autophagy to inhibit tumorigenesis and progression of epithelial ovarian carcinoma by regulating activity of ATG3. Oncotarget 8: 31714-31725, 2017.

30. Scaletta G, Plotti F, Luvero D, Capriglione S, Montera R, Miranda A, Lopez S, Terranova C, De Cicco Nardone C and Angioli R: The role of novel biomarker HE4 in the diagnosis, prognosis and follow-up of ovarian cancer: A systematic review. Expert Rev Anticancer Ther 17: 827-839, 2017.

31. Resnick KE, Alder H, Hagan JP, Richardson DL, Croce CM and Cohn DE: The detection of differentially expressed microRNAs from the serum of ovarian cancer patients using a novel real-time PCR platform. Gynecol Oncol 112: 55-59, 2009.

32. Kan CW, Hahn MA, Gard GB, Maidens J, Huh JY, Marsh DJ and Howell VM: Elevated levels of circulating microRNA-200 family members correlate with serous epithelial ovarian cancer. BMC Cancer 12: 627, 2012

33. Guo F, Tian J, Lin Y, Jin Y, Wang L and Cui M: Serum microRNA-92 expression in patients with ovarian epithelial carcinoma. J Int Med Res 41: 1456-1461, 2013.

34. Wang F, Yuan JH, Wang SB, Yang F, Yuan SX, Ye C, Yang N, Zhou WP, Li WL, Li W and Sun SH: Oncofetal long noncoding RNA PVT1 promotes proliferation and stem cell-like property of hepatocellular carcinoma cells by stabilizing NOP2 Hepatology 60: 1278-1290, 2014.

This work is licensed under a Creative Commons Attribution-NonCommercial-NoDerivatives 4.0 International (CC BY-NC-ND 4.0) License. 